Pacific Journal of Mathematics

THE SPECIES OF BORDERED KLEIN SURFACES WITH
MAXIMAL SYMMETRY OF LOW GENUS

COY LEWIS MAY 


\title{
THE SPECIES OF BORDERED KLEIN SURFACES WITH MAXIMAL SYMMETRY OF LOW GENUS
}

\author{
COY L. MAY
}

\begin{abstract}
A compact bordered Klein surface of genus $g \geq 2$ is said to have maximal symmetry if its automorphism group is of order $12(g-1)$, the largest possible. For each value of the positive integer $g$ there are, of course, several different topological types of bordered surfaces of genus $g$; each distinct topological type is called a species of the genus $g$. Here we classify the species of bordered Klein surfaces with maximal symmetry of genus $g \leq 40$; there are 32 species in 18 different genera. We also classify the species with maximal symmetry that have no more than 5 boundary components. To aid in the classification two group-theoretic constructions that give new surfaces with maximal symmetry and a family of $M^{*}$-groups are introduced. We also establish several general results about the species of a surface with maximal symmetry. In particular we show that if $X$ is a non-orientable bordered surface with maximal symmetry and solvable automorphism group, then the genus of $X$ is odd.
\end{abstract}

0. Introduction. Let $X$ be a compact Klein surface [1] of (algebraic) genus $g \geq 2$. Then the group of automorphisms of $X$ is finite, and it is well-known just how large this group can be. The size of the best possible upper bound depends, however, on the topological type of the surface $X$.

If $X$ is orientable and without boundary, then $X$ is a classical Riemann surface and has at most $168(g-1)$ automorphisms (including the orientation-reversing ones). This of course is just twice the bound Hurwitz obtained in his fundamental paper [7]. Recent research has studied the values of $g$ for which these bounds are attained and the structure of the associated automorphism groups. Most work has concentrated on the groups of orientation-preserving automorphisms (for example, see [2] and [13]), but the full groups were considered in [16]. These groups cannot be solvable [13, p. 19]. Hurwitz's bound $84(g-1)$ is attained for infinitely many values of $g$, but these values have not been classified. The first four values are 3,7,14, and 17, and these are the only ones that are not greater than $40[13$, p. 38].

If the surface $X$ is non-orientable but still without boundary, then the order of the automorphism group is at most $84(g-1)$. This case has received a good deal less attention but has been studied in [15] and [5]. 
Again the bound is attained for infinitely many values of the genus $g$, the first two being 7 and 14 [5].

Finally, if $X$ is a bordered Klein surface, then the bound for the size of the automorphism group of $X$ is the much smaller $12(g-1)$ [8]. Intuitively, the requirement that an automorphism $\alpha: X \rightarrow X$ map each boundary component to another boundary component limits the size of the group. A bordered surface for which the bound $12(g-1)$ is attained is said to have maximal symmetry. Bordered surfaces with maximal symmetry have been studied in [8], [9], [4], and [10], and numerous examples are presented there. For instance there are surfaces with maximal symmetry of genera $2,3,4,5,6$, and 9 , and it is interesting that the bound is attained for both orientable and non-orientable surfaces. In this case as well the bound is attained for infinitely many values of the genus g. The associated automorphism groups may be solvable as well as non-solvable.

For each value of the positive integer $g$ there are, of course, several different topological types of bordered surfaces of genus $g$. This is in marked contrast to the two cases in which the surfaces are without boundary. We shall say that each distinct topological type is a species of the genus $g$. We know the species of a bordered surface when we know its genus, its orientability, and the number of its boundary components. The larger the genus is, the greater the number of species that it contains.

The problem of classifying the bordered Klein surfaces with maximal symmetry was first raised in [4, §3], and one general approach to this problem was given there. The presence of different species within a genus gives the problem a good deal of additional interest and complexity. Also in [4] are several constructions that give many species with maximal symmetry of low genus. As the work on [4] was being concluded, we began to wonder about the completeness of these constructions for surfaces of low genus.

Here we classify the species of bordered Klein surfaces with maximal symmetry of genus $g \leq 40$. The number of species with maximal symmetry in this range is somewhat surprising; there are 32 species in 18 different genera. To exhibit these species we need only the constructions of [4] and some easy group-theoretic constructions. To demonstrate that these are the only species with maximal symmetry in the range, we utilize the basic ideas about coverings of Klein surfaces from [4] and [10]. The structure of the automorphism group of a surface with maximal symmetry also proves helpful. Along the way we establish some general results about the species of a surface with maximal symmetry. 
1. Preliminaries. For any Klein surface $X$, let $A(X)$ denote the group of automorphisms of $X$. We assume all surfaces are compact and of genus $g \geq 2$. By a species $X$ we will always mean a species of bordered Klein surface, that is, a surface $X$ together with a fixed dianalytic structure on $X$. Of course the topological surface may carry many different dianalytic structures.

A finite group $G$ is called an $M^{*}$-group [9] if it is generated by three distinct non-trivial elements $t, u$, and $v$ which satisfy the relations

$$
t^{2}=u^{2}=v^{2}=(t u)^{2}=(t v)^{3}=1 .
$$

The order of $u v$ is called an index of $G$. The fundamental result about $M^{*}$-groups is the following.

TheOREM A ([9], [4]). A finite group $G$ is an $M^{*}$-group with index $q$ if and only if $G$ is the automorphism group of a bordered Klein surface $X$ with maximal symmetry and $k$ boundary components, where

$$
o(G)=2 q k \text {. }
$$

In this situation we will frequently say that $G$ acts on $X$ with index $q$. Note that the index of an $M^{*}$-group $G$ determines the number of boundary components but not the orientability of the surface on which $G$ acts.

Theorem A was established using the representation of a Klein surface as a quotient of the upper half-plane $H$ by a non-euclidean crystallographic (NEC) group. In particular, the finite group $G$ is the automorphism group of a bordered surface $X$ with maximal symmetry if and only if there exist a proper NEC group $\Lambda$ with signature $(2,2,2,3)$ and a homomorphism $\phi: \Lambda \rightarrow G$ onto $G$ such that $X=H / \Gamma$, where $\Gamma=$ kernel $\phi$ is a bordered surface group [9, pp. 4-6].

Let $G$ be an $M^{*}$-group with generators $t, u$, and $v$ satisfying the relations (1.1) with $q=o(u v)$. Set $x=t u$ and $z=u v$. Then $G$ is generated by $u, x$, and $z$ which satisfy the relations

$$
u^{2}=x^{2}=z^{q}=(u x)^{2}=(u z)^{2}=(x z)^{3}=1 .
$$

Sometimes it is more convenient to work with these generators; for example, see [10].

Let $X$ and $X^{\prime}$ be bordered Klein surfaces, and let $\phi: X \rightarrow X^{\prime}$ be an unramified normal covering (without folding) of the surface $X^{\prime}$. If every automorphism of $X^{\prime}$ lifts to an automorphism of $X$, then $\phi$ is a full covering. The basic results about full coverings of surfaces with maximal symmetry are the following. 
THEOREM B [4]. Let $\phi: X \rightarrow X^{\prime}$ be a full covering of the bordered Klein surface $X^{\prime}$. If $X^{\prime}$ has maximal symmetry, then so does $X$. In this case, $A\left(X^{\prime}\right)=A(X) / N$, where $N$ is the group of covering transformations.

THEOREM C [4]. Let $X$ be a bordered Klein surface with maximal symmetry, and let $N$ be a normal subgroup of $G=A(X)$ with $[G: N]>6$. Let $X^{\prime}=X / N, G^{\prime}=G / N$, and let $\phi: X \rightarrow X^{\prime}$ be the quotient map. Then $X^{\prime}$ has maximal symmetry with automorphism group $G^{\prime}$ and $\phi$ is a full covering.

An $M^{*}$-group is said to be $M^{*}$-simple [4] if it has no proper $M^{*}$-quotient groups. If $X$ has maximal symmetry and $A(X)$ is $M^{*}$-simple, then we say $X$ has primitive maximal symmetry. The following is an immediate consequence of Theorem $\mathrm{C}$.

THEOREM D [4]. If $X$ has maximal symmetry, then it is a full covering of a surface with primitive maximal symmetry.

Thus the surfaces with primitive maximal symmetry are of fundamental importance in the classification of surfaces with maximal symmetry.

Let $\phi: X \rightarrow X^{\prime}$ be a full covering of degree $d$, and let $C_{t}$ be the $i$ th component of $\partial X$. The local boundary degree $b_{l}$ is the number of times $C_{i}$ wraps around its image in $\partial X^{\prime}$. If all of the $b_{i}$ are equal, then the common value $b$ is called the boundary degree of $\phi$. In general, of course, a covering will not have a global boundary degree. However, it is important to note that if the surface $X^{\prime}$ has maximal symmetry, then the full covering $\phi$ has a well-defined boundary degree $[4, \S 3]$.

Now suppose the full covering $\phi$ has boundary degree $b$. If $k$ and $k^{\prime}$ are the numbers of components of $\partial X$ and $\partial X^{\prime}$ respectively, then

$$
k b=k^{\prime} d,
$$

and clearly the boundary degree $b$ divides $d$. If $b=d$, then we say $\phi$ is a fully wound covering [10], since $\phi$ is a full covering with maximal winding. Some basic results about fully wound coverings of surfaces with maximal symmetry were established in [10]. We shall need the one that relates this geometric concept to the structure of the automorphism group.

Let $X$ be a bordered Klein surface with maximal symmetry, and let $G=A(X)$ have index $q$ and generators $u, x$, and $z$ satisfying the relations (1.2). 
THEOREM E [10]. Let $\phi: X \rightarrow X^{\prime}$ be a full covering of a surface $X^{\prime}$ with maximal symmetry, and let $A\left(X^{\prime}\right)$ have index $m$. Then the covering $\phi$ is fully wound if and only if in the group $G=A(X), x z^{m}=z^{m} x$, and $X^{\prime}=X / M$, where $M=\left\langle z^{m}\right\rangle$.

Let $X$ be a bordered Klein surface with maximal symmetry, and let $G=A(X)$ have generators $t, u$, and $v$ satisfying the relations (1.1). We fix the following notation.

$$
\begin{aligned}
& g=\text { genus of } X ; \\
& p=\text { topological genus of } X ; \\
& k=\text { number of boundary components of } X ; \\
& q=o(u v), \text { the index of } G .
\end{aligned}
$$

We mention one important relation involving these integers.

$$
g= \begin{cases}2 p+k-1 & \text { if } X \text { is orientable } \\ p+k-1 & \text { if } X \text { is non-orientable. }\end{cases}
$$

Also, to indicate the orientability (" $o$ " for short) of a surface in a table, we shall use a "+" if the surface is orientable and a " - " if it is not.

2. Full coverings. Here we establish some facts about full coverings that will be useful later.

Proposition 1. Let $\phi: X \rightarrow X^{\prime}$ be a full covering. If $X^{\prime}$ is orientable, then so is $X$. If $X^{\prime}$ is non-orientable, and the degree of $\phi$ is odd, then $X$ is also non-orientable.

Proof. First let $X^{\prime}$ be orientable. Then since $\partial X$ is not folded along $\partial X^{\prime}$, we can lift the orientation of $X^{\prime}$ to define an orientation of $X$.

Now suppose $X^{\prime}$ is non-orientable, and let $Z$ be the orienting double of $X^{\prime}$ [1, pp. 40-42]. If $X$ were orientable, there would exist a covering $f: X \rightarrow Z$ such that the following diagram commutes.

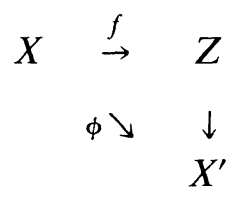

But then the degree of $\phi$ would be twice the degree of $f$, which would not be possible if the degree of $\phi$ were odd. Therefore in this case $X$ is non-orientable. 
If the covering is fully wound, then we can improve this result.

Proposition 2. Let $\phi: X \rightarrow X^{\prime}$ be a fully wound covering. Then $X$ and $X^{\prime}$ are both orientable or both non-orientable.

Proof. If $X^{\prime}$ is orientable, then so is $X$ by Proposition 1.

Let $X^{\prime}$ be non-orientable, and let $Z$ be the orienting double of $X^{\prime}$. If $X$ were orientable, then as in the previous proof there would exist a covering $f: X \rightarrow Z$ such that the diagram (2.1) commutes. But the orienting double $Z$ has $2 k^{\prime}$ boundary components, while $X$ has only $k=k^{\prime}$ since $\phi$ is fully wound. Hence there is no such covering $f$, and $X$ is non-orientable.

The next result concerns surfaces with maximal symmetry.

Proposition 3. Let $X$ and $X^{\prime}$ be surfaces with maximal symmetry, and let $\phi: X \rightarrow X^{\prime}$ be a full covering. Then $k^{\prime}$ divides $k$ and $q^{\prime}$ divides $q$.

Proof. The covering $\phi$ has a well-defined boundary degree $b$ which divides the degree $d$ of the covering. Then $k^{\prime}$ divides $k$ immediately from (1.3). Further, $o(G)=o\left(G^{\prime}\right) \cdot d$ so that $2 q k=2 q^{\prime} k^{\prime} d$. Then using (1.3) we have $q k=q^{\prime} k b$. Thus

$$
q=b q^{\prime}
$$

and $q^{\prime}$ divides $q$.

We shall see later that these three easy propositions have a surprising range of applications.

3. $M^{*}$-groups. The $M^{*}$-groups are closely related to two well-known families of groups. The relationships are hardly surprising in view of the correspondence between surfaces with maximal symmetry and regular maps $[4, \S 6]$.

First let $[n, q]$ denote the group with generators $t, u$, and $v$ and defining relations

$$
t^{2}=u^{2}=v^{2}=(t v)^{n}=(u v)^{q}=(t u)^{2}=1 .
$$

These groups have been investigated extensively [3]. If $G$ is an $M^{*}$-group with index $q$, then $G$ is a quotient of the group $[3, q]$. Further, any finite group $[3, q]$ is an $M^{*}$-group; however, $[3, q]$ is finite only for $q \leq 5[3, \mathrm{p}$. $37]$. At any rate it is an easy matter to classify the $M^{*}$-groups with small index. 
$M^{*}$-groups with index $q \leq 5$

\begin{tabular}{|cccc|lrrr|}
\hline$G$ & $q$ & $r$ & $g$ & \multicolumn{1}{|l}{$G$} & $q$ & $r$ & $g$ \\
$C_{2} \times S_{3} \simeq[3,2]$ & 2 & 6 & 2 & $C_{2} \times S_{4} \simeq[3,4]$ & 4 & 6 & 5 \\
$S_{4} \simeq[3,3]$ & 3 & 4 & 3 & $A_{5}$ & 5 & 5 & 6 \\
$S_{4}$ & 4 & 3 & 3 & $C_{2} \times A_{5} \simeq[3,5]$ & 5 & 10 & 11 \\
\hline
\end{tabular}

Now let $G^{n, q, r}$ be the group with generators $A, B$, and $C$ and defining relations

(3.1) $A^{n}=B^{q}=C^{r}=(A B)^{2}=(B C)^{2}=(C A)^{2}=(A B C)^{2}=1$.

If we set $T=B C, U=C A$, and $V=B C A$, then we obtain the presentation

$$
T^{2}=U^{2}=V^{2}=(T U)^{2}=(T V)^{n}=(U V)^{q}=(T U V)^{r}=1 .
$$

Thus if $G$ is an $M^{*}$-group with $q=o(u v)$ and $r=o(t u v)$, then $G$ is a quotient group of $G^{3, q, r}$. If $q$ and $r$ are not large, this severely limits the possibilities for $G$. Of course any finite group $G^{3, q, r}$ is an $M^{*}$-group. For the complete table of known finite groups $G^{n, q, r}$, see [3, pp. 139, 140].

Next we introduce a family of $M^{*}$-groups that is closely related to the concept of a fully wound covering. These groups are extensions of ones studied by Newman [11, p. 269].

LemMa 1. Suppose $1 \leq n \leq 5$, and let $H_{n}$ be the group with generators $u, x$, and $z$ and defining relations

$$
u^{2}=x^{2}=(u x)^{2}=(u z)^{2}=(x z)^{3}=1, \quad x z^{n}=z^{n} x .
$$

Then $z^{n}$ has order $\alpha_{n}$ and $H_{n}$ is an $M^{*}$-group with index $n \alpha_{n}$, where $\alpha_{n}$ and $o\left(H_{n}\right)$ are given by the following table.

$$
\begin{array}{cccccc}
n & 1 & 2 & 3 & 4 & 5 \\
\alpha_{n} & 6 & 3 & 4 & 6 & 12 \\
o\left(H_{n}\right) & 12 & 36 & 96 & 288 & 1440
\end{array}
$$

Proof. Let $F_{n}$ be the group generated by $x$ and $z$ with defining relations

$$
x^{2}=(x z)^{3}=1, \quad x z^{n}=z^{n} x .
$$

Then both $o\left(z^{n}\right)$ and $o\left(F_{n}\right)$ are known [11, p. 269], and we derive the larger group $H_{n}$ from $F_{n}$. 
Let $\theta: F_{n} \rightarrow F_{n}$ be the automorphism of $F_{n}$ defined by

$$
\theta(x)=x, \quad \theta(z)=z^{-1} .
$$

Now adjoin to $F_{n}$ a new element $u$ of order two which transforms the elements of $F_{n}$ according to the automorphism $\theta$. The order of the larger group is twice that of $F_{n}$, and it is defined by the relations (3.2) together with

$$
u x u=x, \quad u z u=z^{-1}, \quad u^{2}=1 .
$$

The new group is $H_{n}$, of course, and the entries in the table follow from the corresponding facts about $F_{n}\left[11\right.$, p. 269]. Clearly $H_{n}$ is an $M^{*}$-group with index $o(z)=n \alpha_{n}$.

Lemma 2. Suppose $1 \leq n \leq 5$ and $m$ divides $\alpha_{n}$. Let $H_{n, m}$ be the group with generators $u, x$, and $z$ and defining relations

$$
u^{2}=x^{2}=(u x)^{2}=(u z)^{2}=(x z)^{3}=z^{n m}=1, \quad x z^{n}=z^{n} x .
$$

Then $o\left(H_{n, m}\right)=m \cdot o\left(H_{n}\right) / \alpha_{n}$.

Proof. Fix the integer $m$. Let $D$ be the subgroup of $H_{n}$ generated by $z^{n m}$. Then it is easy to see that $D$ is normal in $H_{n}$, and clearly $H_{n, m}=$ $H_{n} / D$. But $o(D)=o\left(z^{n}\right) / m=\alpha_{n} / m$. Thus $o\left(H_{n, m}\right)=o\left(H_{n}\right) / o(D)=$ $m \cdot o\left(H_{n}\right) / \alpha_{n}$.

If $n=1$ and $m \leq 3$, then $H_{n, m}$ is not an $M^{*}$-group, but in general $H_{n, m}$ is an $M^{*}$-group with index $n m$.

Note that for each value of $n \geq 2$ we have a family of fully wound coverings. For example, each surface with maximal symmetry on which a group $H_{4, m}$ acts is, by Theorem E, a fully wound covering of a surface of genus 5 on which the smallest group $H_{4,1}$ acts. These families of surfaces with maximal symmetry were obtained in an entirely different way in [4, §4].

The importance of the groups $H_{n, m}$ in the classification of the species with maximal symmetry is indicated by the following result from [10, $\mathrm{p}$. 27]. Let $X$ be a bordered Klein surface with maximal symmetry, topologically different from the projective plane with three holes (a very special species). Let $G=A(X)$ have index $q$ and generators $u, x$, and $z$ satisfying the relations (1.2).

THEOREM F [10]. There is a positive integer $n \leq k$ such that $n$ divides $q$ and $x z^{n}=z^{n} x$. Further, the subgroup $N=\left\langle z^{n}\right\rangle$ is normal in $G$. 
If it happens that $n \leq 5$, then the generators $u, x$, and $z$ satisfy the relations (3.3). This means $G$ is a quotient of the group $H_{n, m}$ where $n m=q$. Since this will always be the case when $k \leq 5$, the groups $H_{n, m}$ can be used to classify the species with maximal symmetry with no more than 5 boundary components. These groups and Theorem $F$ easily yield the following.

Proposition 4. If $k \leq 5$, then $k$ divides $\gamma_{n}$ for some $n \leq k$, where $\gamma_{n}$ is given by the following table.

$$
\begin{array}{cccccc}
n & 1 & 2 & 3 & 4 & 5 \\
\gamma_{n} & 1 & 3 & 4 & 6 & 12
\end{array}
$$

Proof. We know that for some $n \leq k, G$ is a quotient of the group $H_{n, m}$ where $n m=q$. Then $o\left(H_{n, m}\right)=t \cdot o(G)$ for some integer $t$. Now $o(G)=2 q k=2 m n k$, and using Lemma 2, we have $2 n m k t=m$. $o\left(H_{n}\right) / \alpha_{n}$, or $k t=o\left(H_{n}\right) /\left(2 n \alpha_{n}\right)$. Set $\gamma_{n}=o\left(H_{n}\right) /\left(2 n \alpha_{n}\right)$. Then $k$ divides $\gamma_{n}$, and the values of $\gamma_{n}$ follow from Lemma 1 .

COROLlaRY. There are no bordered Klein surfaces with maximal symmetry with $k=2$ or $k=5$.

It is not hard to finish the classification, and we have our first main result.

THEOREM 1. The species of bordered Klein surfaces with maximal symmetry with $k \leq 5$ are the following.

\begin{tabular}{|llll|llll|}
\hline$g$ & $p$ & $o$ & $k$ & $g$ & $p$ & $o$ & $k$ \\
\hline 2 & 1 & + & 1 & 3 & 0 & + & 4 \\
2 & 0 & + & 3 & 5 & 1 & + & 4 \\
3 & 1 & - & 3 & 9 & 3 & + & 4 \\
4 & 1 & + & 3 & & & & \\
\hline
\end{tabular}

Proof. There exists a Klein surface of each species in the table that has maximal symmetry $[4, \S \S 2$ and 4]. Further, the species with $k=1$ is unique $[4, \S 2]$. 
Let $X$ have maximal symmetry with $k \leq 5$ and let $G=A(X)$. We know that $k \neq 2$ and $k \neq 5$. Assume that topologically $X$ is not the projective plane with 3 holes. Then there is an integer $n \leq k$ such that $k$ divides $\gamma_{n}$ and $G$ is a quotient group of $H_{n, m}$, where $n m=q$.

Suppose $k=4$. Then immediately $n=3$ by Proposition 4 . Note that $o\left(H_{3, m}\right)=24 m$. But $o(G)=2 q k=2 \cdot 3 m \cdot 4=24 m$ as well. Thus $G \simeq$ $H_{3, m}$ where $3 m=q$, and $G$ acts on $X$ with the presentation (3.3). The possibilities for $m$ are 1,2 , and 4 . If $m=1$, then $q=3$ and $g=3$. Then by (1.4) $X$ is orientable and $p=0$. If $m=2$, then $q=6, g=5$, and $X$ is a fully wound covering of a surface with genus 3 and $k=4$. Hence $X$ is orientable by Proposition 2 and $p=1$ by (1.4). Finally, if $m=4$, then $q=12, g=9, X$ is orientable (as with $m=2$ ), and $p=3$.

The proof for $k=3$ is similar and is omitted.

4. Constructions. In this section we give the constructions we need to exhibit the species with maximal symmetry of genus $g \leq 40$. The first four constructions were obtained in [4, Theorems $9,10,12$, and 13] using the theory of covering spaces and the fundamental group. Here we introduce notation for each construction. Let $X$ be a bordered Klein surface.

Construction 1. Let $X$ be orientable, and let $m$ and $n$ be positive integers such that $m$ divides $n$. Then there is a full cover $S_{n}^{m}(X)$ of $X$ with degree $d=n^{2 p} \cdot m^{k-1}$ and boundary degree

$$
b=\left\{\begin{array}{cc}
m & \text { if } k>1 \\
1 & \text { if } k=1
\end{array}\right.
$$

Further, the surface $S_{n}^{m}(X)$ is orientable.

Construction 2. Let $X$ be orientable, and let $m$ and $n$ be positive integers such that $m$ divides $(k, n)$. Then there is a full cover $R_{n}^{m}(X)$ of $X$ with degree $d=n^{2 p} \cdot m$ and boundary degree $b=m$. Further, the surface $R_{n}^{m}(X)$ is orientable.

Construction 3. Let $X$ be non-orientable with $k>1$, and let $m$ and $n$ be positive integers such that $m$ divides $n$. Then there is a full cover $S_{n}^{m}(X)$ of $X$ with degree $d=n^{p-1} \cdot m^{k-1} \cdot(2 m, n)$ and boundary degree $b=m$. The surface $S_{n}^{m}(X)$ is orientable if and only if $n$ is even. 
Construction 4. Let $X$ be non-orientable, and let $n$ be an even positive integer. Then there is a full cover $R_{n}(X)$ with boundary degree

$$
b= \begin{cases}(4, n) / 2 & \text { if } k \text { is odd } \\ 2 & \text { if } k \text { is even }\end{cases}
$$

and degree $d=2 b n^{p-1}$. Further, the surface $R_{n}(X)$ is orientable.

Now let $X$ have maximal symmetry, and suppose a full cover $Z$ of $X$ has been obtained by one of these constructions. Then $Z$ has maximal symmetry by Theorem $\mathrm{B}$, and it is easy to determine the species of $Z$. First, if $\gamma$ is the genus of $Z$, then $\gamma-1=d(g-1)$. Then we can calculate the number of components of $\partial Z$ using (1.3) and the topological genus of $Z$ from (1.4).

The following result from [10, p. 24] gives a useful method for finding a second index for an $M^{*}$-group.

Proposition 5. Let $G$ be an $M^{*}$-group with generators $t$, $u$, and $v$ satisfying the relations (1.1) and index $q=o(u v)$. If $r=o($ tuv $)$, then $G$ also has index $r$.

Proposition 5 was established by noting that $t, u^{\prime}=t u$, and $v$ are another set of generators that satisfy the relations (1.1). Of course determining $o(t u v)$ might be troublesome without a full presentation for the $M^{*}$-group $G$.

Suppose $X$ has maximal symmetry and the $M^{*}$-group $G$ acts on $X$ with index $q$. If $r=o(t u v) \neq q$, then there is another species $Y$ of the same genus that has maximal symmetry and of course $G=A(Y)$. To construct the Klein surface $Y$, just introduce the generators $t, u^{\prime}$, and $v$ and then apply Theorem A. We shall denote the new surface $Y$ by $\operatorname{Op}(X)$. The notation was suggested by a construction of Wilson [17, p. 562] on regular maps. We emphasize that the notation does not imply that $\mathrm{Op}(X)$ is a cover of $X$, which it cannot be. The number $h$ of components of $\partial Y$ can be found from $o(G)=2 r h$, but the orientability of $Y$ remains to be determined.

EXAMPLE. Let $W$ be a torus with three holes that has maximal symmetry, and let $X=S_{3}^{1}(W)$. $X$ is a surface with $g=28, p=1, k=27$, and $G=A(X)$ has order 324 and index $q=6$. Further, $G$ has a normal subgroup $N$ of order 9 such that $X / N=W$. Let $r=o($ tuv $)$ so that $G$ is a quotient of the group $G^{3,6, r}$. Since the order of $G^{3,6,6}$ is 108 [3, p. 139], we 
know $r>6$. Hence $Y=\mathrm{Op}(X)$ is a different species of genus 28 with maximal symmetry. Since the subgroup $N$ is normal in $G=A(Y), Z=$ $Y / N$ is a surface with maximal symmetry of genus 4 and the quotient map $\phi: Y \rightarrow Z$ is a full covering. But there is only one species of genus 4 with maximal symmetry, by (1.4) and Theorem 1 . Topologically $Z$ is a torus with three holes. Now $Y$ is orientable by Proposition 1. We still need to determine $r$, the index of $A(Y)$. Since $A(Z)$ has index 6, we have $r=6 b$ from (2.2), where $b$ is the boundary degree of the covering $\phi$. Further, $b$ divides 9 , the degree of $\phi$. Thus $r=18$ or $r=54$. If $r=54$, then $Y$ would have 3 boundary components, contradicting Theorem 1 . Hence $r=18$, and $Y=\mathrm{Op}(X)$ is an orientable surface with 9 boundary components and topological genus 10. It is interesting that this surface cannot be explained by the constructions of $[4, \S 4]$.

The remaining two constructions are group-theoretic in nature. We shall only need the notions of direct and semi-direct products and the following general idea. Let $X$ have maximal symmetry, and let $G=A(X)$ have generators $t, u$, and $v$ satisfying the usual relations (1.1). Suppose that from $G$ we construct a larger $M^{*}$-group $G^{*}$ with generators $t^{*}, u^{*}$, and $v^{*}$ satisfying the relations (1.1) and that there is a homomorphism $\pi: G^{*} \rightarrow G$ defined by $\pi\left(t^{*}\right)=t, \pi\left(u^{*}\right)=u$, and $\pi\left(v^{*}\right)=v$. Let $K=$ kernel $\pi$ so that $G^{*} / K=G$, and represent $X$ in the form $X=H / \Gamma$, where $\Gamma$ is a bordered surface group. Then there exist a proper NEC group $\Lambda$ with signature $(2,2,2,3)$ and a homomorphism $\phi: \Lambda \rightarrow G$ onto $G$ such that kernel $\phi=\Gamma$. By considering the action of $\phi$ on the generators of $\Lambda$ (the image of each generator is either $t, u, v$, or 1$)[9, \mathrm{p} .6]$, it is easy to see that $\phi$ will factor through $\pi$, that is, there is a homomorphism $\alpha: \Lambda \rightarrow G^{*}$ onto $G^{*}$ such that $\pi \circ \alpha=\phi$. Further, $\Delta=$ kernel $\alpha$ is a bordered surface group. Then the $M^{*}$-group $G^{*}$ acts on the surface $Z=H / \Delta$. Clearly $\Delta \subset \Gamma$ and $\Gamma / \Delta=K$. Finally we have

$$
Z / K=(H / \Delta) /(\Gamma / \Delta)=H / \Gamma=X .
$$

Thus $Z$ is a full covering of $X$.

THEOREM 2. Let $X$ have maximal symmetry. If $G=A(X)$ has odd index $q$, then $C_{2} \times G$ is an $M^{*}$-group with index $2 q$ and there is a surface $Q(X)$ with maximal symmetry that is a fully wound double covering of $X$. Further, $Q(X)$ is orientable if and only if $X$ is.

Proof. Let $C_{2}=\langle a\rangle$, and choose $t^{*}=(a, t), u^{*}=(1, u)$, and $v^{*}=$ $(a, v)$. Then $t^{*}, u^{*}$, and $v^{*}$ generate $C_{2} \times G$ and satisfy the relations (1.1) with $o\left(u^{*} v^{*}\right)=2 q$. Let $\pi$ be the obvious homomorphism $\pi: C_{2} \times G \rightarrow G$, 
and note that $\pi\left(t^{*}\right)=t, \pi\left(u^{*}\right)=u$, and $\pi\left(v^{*}\right)=v$. Then, as above, obtain the surface $Z$ on which $C_{2} \times G$ acts. Since the index of $C_{2} \times G$ is $2 q, Z$ and $X$ have the same number of boundary components. Thus $Z$ is a fully wound covering of $X$. Finally, $Z$ is orientable if and only if $X$ is, by Proposition 2. Henceforth we denote this double covering $Z$ by $Q(X)$.

The construction will not work if the index $q$ is even. If $X$ is non-orientable, then $Q(X)$ is not the orienting double of $X$ and $X$ has two double covers with maximal symmetry. Each has automorphism group $C_{2} \times G$.

EXAMPLE. Let $X$ be a non-orientable surface of genus 6 with primitive maximal symmetry, so that $A(X) \simeq A_{5}, q=5, p=1$, and $k=6$. Then $Q(X)$ is a non-orientable surface of genus 11 with maximal symmetry that has topological genus 6 and 6 boundary components. This is another species that cannot be explained by the constructions of $[4, \S 4]$ (but see $[4$, $\S 6])$.

Now consider the subgroup $H=\langle t u, u v\rangle$ of the $M^{*}$-group $G$. The index of $H$ in $G$ is at most two. Suppose $[G: H]=2$. This will always be the case when $X$ is orientable, since $u$ is a reflection. Then let $\theta$ be the quotient map

$$
\theta: G \rightarrow G / H \simeq C_{2}=\operatorname{Aut}\left(C_{3}\right)
$$

and construct the semi-direct product $C_{3} \times{ }_{\theta} G$ (using the notation of [12]).

THEOREM 3. Let $X$ have maximal symmetry. If $[G: H]=2$ and $(3, q)$ $=1$, then $C_{3} \times{ }_{\theta} G$ is an $M^{*}$-group with index $3 q$ and there is a surface $T(X)$ with maximal symmetry that is a fully wound triple covering of $X$. Further $T(X)$ is orientable if and only if $X$ is.

Proof. Let $C_{3}=\langle b\rangle$, and choose $t^{\prime}=(b, t), u^{\prime}=(b, u)$, and $v^{\prime}=$ $(1, v)$. Then $t^{\prime}, u^{\prime}$, and $v^{\prime}$ generate $C_{3} \times_{\theta} G$ and satisfy the relations (1.1) with $o\left(u^{\prime} v^{\prime}\right)=3 q$ and $o\left(t^{\prime} u^{\prime} v^{\prime}\right)=r=o(t u v)$. Let $\pi$ be the obvious homomorphism $\pi: C_{3} \times{ }_{\theta} G \rightarrow G$, and note that $\pi\left(t^{\prime}\right)=t, \pi\left(u^{\prime}\right)=u$, and $\pi\left(v^{\prime}\right)$ $=v$. As before obtain the surface $W$ on which the new $M^{*}$-group $C_{3} \times{ }_{\theta} G$ acts. Then $W$ is a fully wound triple covering of $X$. Now denote $W$ by $T(X)$.

Theorem 3 and Proposition 5 can sometimes be combined to yield an additional species with maximal symmetry. 
EXAMPLE. Let $X$ be a sphere with 12 holes that has maximal symmetry, so that $g=11$ and $G=A(X)=C_{2} \times A_{5}$ has index $q=5$ and $r=$ $o($ tuv $)=10$. Then $G^{*}=C_{3} \times_{\theta} G$ is an $M^{*}$-group of order 360 with $q=15$ and $r=10 . G^{*}$ acts on two species of genus 31 with maximal symmetry. $W=T(X)$ is an orientable surface with $p=10$ and $k=12$, while $\mathrm{Op}(W)$ is non-orientable and has $p=14$ and $k=18$.

5. Results used in the search. In this section we collect the results we need to classify the species of bordered Klein surfaces with maximal symmetry of genus $g \leq 40$. We begin by finding all $M^{*}$-simple groups of order at most $468=12(40-1)$. The solvable $M^{*}$-simple groups have already been classified; the only ones are $C_{2} \times S_{3}$ and $S_{4}[4, \S 5]$.

We look first at the simple groups (of composite order). The ones in the range are $A_{5}$ of order $60, \mathrm{PSL}_{2}(7)$ of order 168, and $A_{6}$ of order 360 . These three groups are well-known. A consideration of the possible orders for elements in these groups, together with a check of the finite groups $G^{3, q, r}$, yields the following.

Lemma 3. The only simple $M^{*}$-group of order less than 480 is $A_{5}$, which has index 5.

A simple $M^{*}$-group is, of course, $M^{*}$-simple. If $G$ is a simple $M^{*}$-group, then $G$ acts only on non-orientable surfaces with maximal symmetry, since otherwise the orientation-preserving automorphisms would form a subgroup of index two in $G[8$, p. 206].

Next we search for $M^{*}$-simple groups among the non-solvable groups of order less than 480 . We look first at the groups involving $A_{5}$.

LEMMA 4. There are no $M^{*}$-simple groups of order $60 n$ for $2 \leq n \leq 7$.

Proof. For $n \neq 4$ this follows easily from the list of non-solvable groups of these orders in [6, pp. 417, 418].

Let $G$ be a non-solvable group of order $240=16 \cdot 3 \cdot 5$, and let $S$ be a Sylow 5-subgroup of $G$. Since $G$ is non-solvable, $S$ is not normal in $G$. Let $n_{5}$ be the number of Sylow 5-subgroups of $G$, and let $N$ and $C$ be the normalizer and centralizer of $S$ in $G$. Then immediately $n_{5}=6$ or $n_{5}=16$. If $n_{5}=16$, then $o(N)=15$ and $N$ would be cyclic. This would force $N=C$, and $S$ would have a normal complement in $G[14$, p. 137]. But then $G$ would be solvable. Therefore $n_{5}=6$.

Now $[G: N]=6$, and there is a homomorphism $\rho: G \rightarrow S_{6}$ such that $K=\operatorname{kernel} \rho \subset N[12$, p. 48]. If $K=N$, then $G$ would be solvable. Since 
$S_{6}$ does not have a subgroup of index $3, K \neq\{1\}$. Thus $K$ is a non-trivial normal subgroup of index larger than 6 , and $G$ is not an $M^{*}$-simple group.

We still need to examine the groups in the range involving $\operatorname{PSL}_{2}(7)$.

LEMMA 5. The only non-solvable $M^{*}$-group of order 336 is $\mathrm{PGL}_{2}(7)$, which has indices 7 and 8.

Proof. Let $G=\mathrm{PGL}_{2}(7)$. Then $G \simeq G^{3,7,8} \simeq G^{3,8,7}[3$, p. 139], so that $G$ is an $M^{*}$-group with indices 7 and 8 . An easy consideration of the orders of elements of $G$ and the groups $G^{3, q, r}$ shows that no other index is possible.

The other two non-solvable groups of order 336 have a normal subgroup of order two and quotient $\operatorname{PSL}_{2}(7)$. Therefore these two groups are not $M^{*}$-groups, since $\operatorname{PSL}_{2}(7)$ is not.

$\mathrm{PGL}_{2}(7)$ is clearly $M^{*}$-simple. We need to determine the species on which $G=\mathrm{PGL}_{2}(7)$ acts. First let $G$ act on $X$ with index $q=8$, so that $k=21$. Since $g=29, X$ is non-orientable of topological genus $p=9$ just from (1.4).

Next let $G$ act on a surface $Y$ with index $q=7$, so that $k=24$. Suppose for the moment that $Y$ is non-orientable, and let $Y_{0}$ be the orienting double of $Y$. Then $Y_{0}$ is of genus $2 g-1=57$, topological genus 5 , and has $2 k=48$ boundary components. Further, $G$ acts as a group of orientation-preserving automorphisms of $Y_{0}\left[1\right.$, pp. 40-43]. Now embed $Y_{0}$ in a surface $M$ without boundary of the same topological genus so that every automorphism of $Y_{0}$ extends to an automorphism of $M$ [4, Th.D]. Thus $G$ acts as a group of orientation-preserving automorphisms of $M$, a Riemann surface of genus 5. But $o(G)=336=84(5-1)$, and the bound of Hurwitz is not attained for surfaces of genus $5[16, p .26]$. Therefore the bordered Klein surface $Y$ is orientable and of topological genus $p=3$.

In summary we state our next main result.

THEOREM 4. The seven species of bordered Klein surfaces with primitive maximal symmetry of genus $g \leq 40$ are the following.

\begin{tabular}{|ccccc|rcccc|}
\hline$g$ & $p$ & $o$ & $k$ & $G$ & $g$ & $p$ & $o$ & $k$ & $G$ \\
\hline 2 & 0 & + & 3 & $C_{2} \times S_{3}$ & 6 & 1 & - & 6 & $A_{5}$ \\
2 & 1 & + & 1 & $C_{2} \times S_{3}$ & 29 & 3 & + & 24 & $\mathrm{PGL}_{2}(7)$ \\
3 & 0 & + & 4 & $S_{4}$ & 29 & 9 & - & 21 & $\mathrm{PGL}_{2}(7)$ \\
3 & 1 & - & 3 & $S_{4}$ & & & & & \\
\hline
\end{tabular}


Let $X$ be any bordered surface with maximal symmetry of genus $g$. If $g \leq 40$, then by Theorem $\mathrm{D} X$ is a full cover of one of these seven species. Also, if $A(X)$ is solvable (with no restriction on the size of $g$ ), then $X$ is a full cover of one of the four species of genus 2 or 3 . These two observations and the results of $\$ 2$ place effective restrictions on the species of $X$. For example, we easily obtain the following surprising result.

THEOREM 5. If $X$ is a non-orientable bordered surface with maximal symmetry such that $G=A(X)$ is solvable, then

(1) the genus $g$ of $X$ is odd,

(2) 3 divides $k$, and

(3) 4 divides $q$.

Proof. By Proposition 1, $X$ must be a full cover of a projective plane with three holes, the only non-orientable species with primitive maximal symmetry and solvable automorphism group. Then 3 divides $k$ and 4 divides $q$ by Proposition 3, and 24 divides $o(G)=12(g-1)$ by Theorem C so that $g$ is odd.

Of course, for many (even) values of $g$, all groups of order $12(g-1)$ are solvable. Non-orientable surfaces with maximal symmetry of even genus do exist; there is one of genus 6 for instance.

Next we establish three general results that are useful in classifying species with maximal symmetry. The first concerns those with $k<q$.

Proposition 6. Let $X$ be a bordered Klein surface with maximal symmetry and $k>3$. If $k<q$, then $X$ is a fully wound covering of a surface $X^{\prime}$ with maximal symmetry of lower genus.

Proof. Let $G=A(X)$ have generators $u, x$, and $z$ satisfying (1.2) and apply Theorem $\mathrm{F}$. There is a positive integer $n \leq k$ such that $n$ divides $q$ and $x z^{n}=z^{n} x$. The subgroup $N=\left\langle z^{n}\right\rangle$ is normal in $G$, and $[G: N]=$ $2 n k>6$ since $k>3$. Let $X^{\prime}=X / N$ and $G^{\prime}=G / N$. Then by Theorem C, $X^{\prime}$ has maximal symmetry, and the covering $X \rightarrow X^{\prime}$ is fully wound by Theorem E. Since $o\left(G^{\prime}\right)=2 n k<2 q k=o(G)$, we have $g^{\prime}<g$.

Thus if there is a species $X$ with maximal symmetry and $k<q$, there must be another species $X^{\prime}$ of lower genus with the same number of boundary components. Further, $X^{\prime}$ is orientable if and only if $X$ is, by Proposition 2.

The second result is similar but deals with a different type of surface. 
Proposition 7. Let $X$ be a non-orientable surface with maximal symmetry of genus $g>3$, and let $G=A(X)$ be solvable of order $24 n$. If $n$ is not a power of a prime, then $X$ is a full covering of a non-orientable surface $X^{\prime}$ with maximal symmetry of genus $g^{\prime}$, where $3<g^{\prime}<g$.

Proof. As in the proof of Theorem 5, $X$ is a full cover of a projective plane $\Phi$ with 3 holes. Let $N$ be a normal subgroup of $G$ such that $X / N=\Phi$, and $G / N=A(\Phi) \simeq S_{4}$. Let $M$ be a minimal normal subgroup of $G$ such that $M \subset N$. Since $G$ is solvable, the order of $M$ must be a power of a prime [12, p. 112], so $M \neq N$. Now let $X^{\prime}=X / M$. Then $X^{\prime} /(N / M)=(X / M) /(N / M)=X / N=\Phi$, and the following diagram of quotient maps commutes.

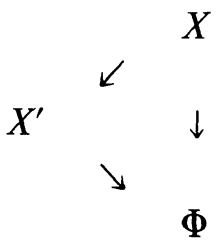

The surface $X^{\prime}$ is non-orientable by Proposition 1 and clearly $3<g^{\prime}<g$.

Like Theorem 5 the next result says that in some genera none of the non-orientable species have maximal symmetry.

THEOREM 6. There are no non-orientable bordered surfaces with maximal symmetry of genus $g=2^{m}+1$ for any integer $m \geq 2$.

Proof. Suppose, to the contrary, such surfaces do exist, and let $X$ be one such surface of the lowest genus, that is, the genus of $X$ is $2^{l}+1$ for some integer $l$ and there are no non-orientable surfaces with maximal symmetry of genus $2^{m}+1$ for any integer $m$ such that $2 \leq m<l$. Since $o(G)=12 \cdot 2^{l}=3 \cdot 2^{l+2}, G$ is solvable by a theorem of Burnside [14, p. 334]. Then 3 divides $k$ and 4 divides $q$ by Theorem 5 .

First we show that $l$ is not 2 or 3 . Suppose $g=5$, so $p+k=6$ by (1.4). Then $k=3$ is the only possibility, but this would contradict Theorem 1 . Therefore $g \neq 5$ and $l \neq 2$. Next suppose $g=9$, so $o(G)=96$ and $q k=48$. Then $k=6$ and $q=8$, since $k \neq 3$ by Theorem 1. By Proposition $6 X$ is a fully wound covering of a surface $X^{\prime}$ with $k^{\prime}=6$ and $g^{\prime}<g$. Moreover, $X^{\prime}$ is non-orientable and $o\left(G^{\prime}\right)$ divides $o(G)$. Thus $o\left(G^{\prime}\right) \leq 48$ and $g^{\prime} \leq 5$. But there is no such surface $X^{\prime}$ just by (1.4). Hence $g \neq 9$ and $l \neq 3$. 
Now we know that $l \geq 4$. As in the preceding proof, let $N$ be a normal subgroup of $G$ such that $G / N \simeq S_{4}$. Then $N$ is a 2-group of order at least 8 , and clearly $N$ must be a minimal normal subgroup of $G$, for otherwise $X$ would be a full cover of another non-orientable surface of lower genus of the form $2^{m}+1$. Therefore $N$ is an elementary abelian 2-group [12, p. 112]. Let $\phi: G \rightarrow S_{4}$ be the quotient map, and let $f \in G$. Since $\phi(f) \in S_{4}$ and $N$ is elementary abelian, $f$ has order $1,2,3,4,6$, or 8 . Hence the $M^{*}$-group $G$ must be a quotient of a group $G^{3, q, r}$, where $q$ and $r$ are elements of the set $\{1,2,3,4,6,8\}$. Since $o(G) \geq 192$ and $G$ is solvable, an examination of the table of groups $G^{n, q, r}\left[3\right.$, p. 139] shows that $G \simeq G^{3,8,6}$, a group of order 192. But it is clear from the presentation (3.1) that $G^{3,8,6}$ has $G^{3,2,6}$ as a quotient group. Since $G^{3,2,6} \simeq C_{2} \times S_{3}$ [3, p. 139], $G$ has a normal subgroup $H$ such that $o(G / H)=12$ and $X / H$ is a surface of genus 2 with maximal symmetry. Therefore $X / H$ is orientable. But then $X$ would also be orientable, by Theorem $\mathrm{C}$ and Proposition 1. This contradicts the assumption that $X$ is non-orientable. Thus there are no non-orientable surfaces with maximal symmetry of genus $2^{m}+1$ for $m \geq 2$.

Finally we establish a result that proves very helpful in eliminating possible orders for $M^{*}$-groups.

THEOREM 7. Let $G$ be a finite group. Let $p$ be a prime larger than 3 such that $p$ divides $o(G)$, and let $S$ be a Sylow p-subgroup of $G$. If $S$ is cyclic and normal in $G$, then $G$ is not an $M^{*}$-group.

Proof. Let $\operatorname{Aut}(S)$ denote the group of automorphisms of $S$. Since $S$ is cyclic, $\operatorname{Aut}(S)$ is abelian [14, p. 120]. Now let $C$ be the centralizer of $S$ in $G$. Since $S$ is normal in $G, G / C$ is isomorphic to a subgroup of $\operatorname{Aut}(S)$ $\left[14\right.$, p. 50]. Hence the commutator subgroup $G^{\prime} \subset C$.

Now suppose $G$ is an $M^{*}$-group. Then $\left[G: G^{\prime}\right]$ divides 4 and $\left[G^{\prime}: G^{\prime \prime}\right]$ divides $9[4, \S 5]$. Now $S$ is the Sylow $p$-subgroup of $G^{\prime}$, and $S$ is central in $G^{\prime}$. By a theorem of Burnside [14, p. 137], $S$ has a normal complement in $G^{\prime}$. Hence $S$ is a factor group of $G^{\prime}$ and $p$ divides $\left[G^{\prime}: G^{\prime \prime}\right]$, a contradiction. Therefore $G$ is not an $M^{*}$-group.

The proofs of the following applications of the theorem are quite similar, and we provide only one.

COROLlaRY 1 [10]. There are no $M^{*}$-groups of order $12 p$ for any prime $p>5$. 
COROLlaRY 2. There are no solvable $M^{*}$-groups of order $24 p, 48 p$, or $72 p$ for any prime $p>3$.

COROLlaRY 3. There are no solvable $M^{*}$-groups of order $12 \mathrm{pr}$ for any primes $p$ and $r$ such that $3 \leq p<r$.

Proof. Suppose $G$ is a solvable $M^{*}$-group of order $12 p r$. Then $G$ has a normal subgroup $H$ of order $p r$ such that $G / H=C_{2} \times S_{3}$. The Sylow $r$-subgroup of $H$ is characteristic in $H$ and hence normal in $G$. But this contradicts the theorem. Thus there are no solvable $M^{*}$-groups of order $12 \mathrm{pr}$.

6. General outline of the search. We began by applying the constructions of $\S 4$ to the species with primitive maximal symmetry of genus $g \leq 40$. In some cases the constructions could be iterated to produce additional species with maximal symmetry in the range. We were also able to discover 3 species with maximal symmetry by using Proposition 5 . Altogether we found 32 species in 18 different genera.

The next step was to eliminate all other possible values in the range for the genus of a surface with maximal symmetry. Here the corollaries to Theorem 7 and the results of $\$ 5$ were surprisingly effective; all possibilities for $g$ except $g=7$ and $g=19$ were quickly eliminated. These two values were easily ruled out by examining groups of orders 72 and 216 and using the results of $[4, \S 5]$ on the commutator subgroups of $M^{*}$-groups. At this stage we knew that the bound $12(g-1)$ was attained for exactly 18 values of $g$ between 2 and 40 .

To classify the species with maximal symmetry we began at $g=2$ and worked upward through these values. Thus at any point all species with maximal symmetry of lower genus were known. In addition, the species with primitive maximal symmetry had already been classified. It proved convenient to consider the species with solvable automorphism group separately.

Suppose the bound $12(g-1)$ is attained for some particular value of $g>3$ in the range, and assume the associated automorphism group is solvable. Then either Theorem 5, Theorem 6, or Proposition 7 immediately eliminates all non-orientable species. The next step is to list the arithmetic possibilities for $q, k$, and $p$ satisfying $2 q k=12(g-1)$ and (1.4) for orientable surfaces. Then we note the species with maximal symmetry that appeared in the constructions of $\$ 4$ and eliminate the others. (The general procedure is illustrated for genus 25 in the first 
example of §7.) Some species are quickly ruled out, as the species with $k \leq 5$ (Theorem 1) and the ones of low topological genus [4, §2] are known. The species with $k<q$ are not hard to handle, by using Proposition 6 or Theorem $F$ and Lemma 1 or 2 . In some genera at this point there still remain one or two species to be eliminated. None could have primitive maximal symmetry, of course. The general strategy is to assume the species has maximal symmetry and then use the facts about full coverings from $\$ 2$ and the completed classification in lower genera to arrive at a contradiction. Here the structure of the automorphism group is of ten quite important. Example 2 of $\S 7$ is a typical case.

The classification of the species with non-solvable automorphism group was somewhat easier, for in the range the bound is attained only for $g=6,11,21,29$, and 31 . For $g=6$ and $g=29$ the group is $M^{*}$-simple and the species are determined by Theorem 4 .

Now suppose $X$ is a species with maximal symmetry of genus $g=11$, 21 , or 31 and the automorphism group $G=A(X)$ is non-solvable. Then $G$ is not $M^{*}$-simple, and $G$ has a normal subgroup $N$ such that $G / N \simeq A_{5}$ and $X / N=\Psi$, a real projective plane with 6 holes. By Proposition 3, 5 divides $q$ and 6 divides $k$. If further $X$ is orientable, then the covering $X \rightarrow \Psi$ factors through the orienting double $\Psi_{0}$ :

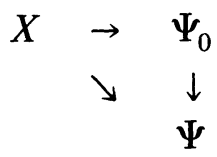

But $\Psi_{0}$ has 12 boundary components, so that in this case 12 must divide $k$. These restrictions are enough to rule out most species in genera 11,21 , and 31 that do not appear in the constructions of $\S 4$. The few remaining species are eliminated using the approach described for the troublesome species with solvable automorphism group. Example 3 treats a fairly routine case.

7. Examples. To indicate the nature of the search we present a few applications of the general theory.

EXAmple 1. $g=25$. First $o(G)=12 \cdot 24=2^{5} \cdot 3^{2}$, so $G$ is solvable. At this stage in the classification we know there are no non-orientable species with maximal symmetry and solvable automorphism group with genus between 3 and 25. Hence, by Proposition 7, there are none of genus 25. Next the arithmetic possibilities for $q, k$ and $p$ for orientable surfaces with $k \geq 6$ are the following. 


\begin{tabular}{|llrr|rrrr|}
\hline & $q$ & $k$ & $p$ & & $q$ & $k$ & $p$ \\
\hline 1. & 24 & 6 & 10 & 4. & 9 & 16 & 5 \\
2. & 18 & 8 & 9 & 5. & 8 & 18 & 4 \\
3. & 12 & 12 & 7 & 6. & 6 & 24 & 1 \\
\hline
\end{tabular}

Species 1 and 3 do have maximal symmetry. Species 6 has topological genus $p=1$ and is eliminated by [4, Th. 3]. Species 2 is ruled out by Proposition 6 since there are no surfaces with maximal symmetry with $k=8$ of lower genus. The remaining two species require special treatment. An easy application of Theorem $F$ and Lemma 1 rules out species 4, while species 5 is eliminated by considering possibilities for the orders of normal 2-subgroups and 3-subgroups of $G$.

EXAmple 2. $g=37, q=9, k=24$. Suppose $X$ has maximal symmetry. Since $o(G)=12 \cdot 36=2^{4} \cdot 3^{3}, G$ is solvable and $X$ is a full cover of one of the four species with maximal symmetry of genus 2 or 3 . By Proposition 3 the only possibility is that $X$ is a full cover of a sphere $\Sigma$ with 4 holes. Then $G$ has a normal subgroup $N$ of order 18 such that $G / N=A(\Sigma) \simeq S_{4}$. Let $M$ be a minimal normal subgroup of $G$ contained in $N$. Since $G$ is solvable, $o(M)$ is a power of a prime, so $o(M)$ is 2, 3, or 9. Then $X^{\prime}=X / M$ is a surface with maximal symmetry of lower genus, and further $k^{\prime}$ divides 24 and $q^{\prime}$ divides 9. By considering the three possibilities for $o(M)$ and using the completed classification in lower genera, we see that there is no such surface $X^{\prime}$. Thus the species $X$ does not have maximal symmetry.

EXAMPLE 3. $g=31, q=15, k=12$. Suppose $X$ has maximal symmetry. Since $o(G)=12 \cdot 30=72 \cdot 5, G$ is not solvable by Corollary 2 to Theorem 7. By Proposition 6, $X$ is a fully wound covering of a surface $X^{\prime}$ with maximal symmetry of lower genus. Since $k^{\prime}=12$, the only possibility is that $X^{\prime}$ is orientable of genus $g^{\prime}=11$. But then $X$ must be orientable by Proposition 2. Indeed the last example of $\$ 4$ contains a surface with maximal symmetry of the orientable species. However, the non-orientable species cannot have maximal symmetry.

8. The species with maximal symmetry. We conclude by listing the species in the range that have maximal symmetry. For each species that does not have primitive maximal symmetry, we also give one method of exhibiting the species as a surface with maximal symmetry. We use the 
constructions of $\S 4$ and the following notational convention. The species will be numbered, and by $X_{i}$ we mean a Klein surface of species $i$ with maximal symmetry.

THEOREM 8. The species of bordered Klein surfaces with maximal symmetry of genus $g \leq 40$ are the following.

\begin{tabular}{|c|c|c|c|c|c|c|c|c|c|c|c|c|c|c|c|c|}
\hline$g$ & $p$ & $o$ & $k$ & & & $g$ & $p$ & $o$ & $k$ & & & $g$ & $p$ & $o$ & $k$ & \\
\hline 1. 2 & 0 & + & 3 & & 12. & 11 & 0 & + & 12 & $S_{2}^{1}\left(X_{8}\right)$ & 23. & 28 & 1 & + & 27 & $S_{3}^{1}\left(X_{5}\right)$ \\
\hline 2. 2 & 1 & + & 1 & & 13. & 11 & 6 & - & 6 & $Q\left(X_{8}\right)$ & 24. & 28 & 10 & + & 9 & $\mathrm{Op}\left(X_{23}\right)$ \\
\hline 3. 3 & 0 & + & 4 & & 14. & 13 & 1 & + & 12 & $S_{2}^{l}\left(X_{5}\right)$ & 25. & 29 & 3 & + & 24 & \\
\hline 4. 3 & 1 & - & 3 & & 15. & 13 & 4 & + & 6 & $R_{3}^{3}\left(X_{6}\right)$ & 26. & 29 & 9 & - & 21 & \\
\hline 5. 4 & 1 & + & 3 & $R_{3}^{3}\left(X_{1}\right)$ & 16. & 17 & 1 & + & 16 & $S_{4}^{4}\left(X_{2}\right)$ & 27. & 31 & 10 & + & 12 & $T\left(X_{12}\right)$ \\
\hline 6. 5 & 0 & + & 6 & $S_{2}^{2}\left(X_{1}\right)$ & 17. & 17 & 3 & + & 12 & $S_{4}^{4}\left(X_{1}\right)$ & 28. & 31 & 14 & - & 18 & $\mathrm{Op}\left(X_{27}\right)$ \\
\hline 7. 5 & 1 & + & 4 & $S_{2}^{2}\left(X_{2}\right)$ & 18. & 21 & 5 & + & 12 & $R_{2}\left(X_{8}\right)$ & 29. & 33 & 5 & + & 24 & $S_{4}^{2}\left(X_{4}\right)$ \\
\hline 8. 6 & 1 & - & 6 & & 19. & 25 & 7 & + & 12 & $\mathrm{Op}\left(X_{20}\right)$ & 30. & 33 & 9 & + & 16 & $R_{2}^{2}\left(X_{7}\right)$ \\
\hline 9. 9 & 2 & + & 6 & $R_{4}\left(X_{4}\right)$ & 20. & 25 & 10 & + & 6 & $T\left(X_{9}\right)$ & 31. & 37 & 1 & + & 36 & $S_{6}^{6}\left(X_{2}\right)$ \\
\hline 10. 9 & 3 & + & 4 & $R_{4}^{4}\left(X_{3}\right)$ & 21. & 26 & 1 & + & 25 & $S_{5}^{5}\left(X_{2}\right)$ & 32. & 37 & 10 & + & 18 & $S_{6}^{6}\left(X_{1}\right)$ \\
\hline 11. 10 & 1 & + & 9 & $S_{3}^{3}\left(X_{1}\right)$ & 22. & 26 & 6 & + & $15^{\circ}$ & $S_{5}^{5}\left(X_{1}\right)$ & & & & & & \\
\hline
\end{tabular}

It should be possible to use our approach to extend the classification to a higher value of $g$. However, the growing number of species per genus indicates the need for new general results.

The data in Theorem 8 for $g \leq 40$ do suggest that the bound $12(g-1)$ is attained rather frequently in the sequence of values of $g$. This is in marked contrast to the situation for Riemann surfaces [13, p. 38]. We also see that species with a wide diversity of values of $p$ and $k$ have maximal symmetry. The number and diversity of these species suggest that classifying all species of bordered Klein surfaces with maximal symmetry may be an enormous problem.

Nevertheless certain pieces of the big problem seem interesting and more approachable. We would like to call attention to the following unsolved problems; also see $[4, \S 3]$.

Few of the surfaces in Theorem 8 are non-orientable, and we already have Theorems 4 and 5 .

Problem 1. Classify the non-orientable species with maximal symmetry and solvable automorphism group.

Some $M^{*}$-groups are supersolvable, e.g. $C_{2} \times S_{3}$ and $S_{3} \times S_{3}$, and these groups are especially tractable.

Problem 2. Find all supersolvable $M^{*}$-groups. 
Problem 3. Classify the species with maximal symmetry and supersolvable automorphism group.

Problem 4 [4, §3]. Given a surface $X$ with primitive maximal symmetry, find all full coverings of $X$, or even all abelian full coverings. In particular, use the theory of covering spaces and the fundamental group as in [4] to explain the coverings $Q(X)$ and $T(X)$.

Problem 5. Determine all possible values for the number $k$ of boundary components of a surface with maximal symmetry. Of course, many sequences of possible values of $k$ are known [4], and $k$ cannot be a prime larger than $3[10$, p. 30]. Still it is not known whether a surface exists for many interesting values of $k$, in particular for $k=8$.

In Theorem 8 there are several genera in which there are 2 species with maximal symmetry but none with 3 .

Problem 6. Determine whether there is a bound (independent of $g$ ) for the number of species within a single genus that can have maximal symmetry. We conjecture that there is not.

\section{REFERENCES}

[1] N. L. Alling and N. Greenleaf, Foundations of the Theory of Klein Surfaces, Lecture notes in Mathematics Vol. 219, Springer-Verlag, Berlin and New York, 1971.

[2] J. M. Cohen, On Hurwitz extensions by PSL 2 (7), Proc. Camb. Phil. Soc., 86 (1979), 395-400.

[3] H. S. M. Coxeter and W. O. J. Moser, Generators and Relations for Discrete Groups, 3rd ed., Ergebnisse der Math. und ihrer Grenzgebiete, Band 14, Springer-Verlag, Berlin and New York, 1972.

[4] N. Greenleaf and C. L. May, Bordered Klein surfaces with maximal symmetry, Trans. Amer. Math. Soc., 274 (1982), 265-283.

[5] W. Hall, Automorphisms and coverings of Klein surfaces, Ph. D. Thesis, University of Southampton (1977).

[6] O. Holder, Bildung zusammengesetzter Gruppen, Math. Ann., 46 (1895), 321-422.

[7] A. Hurwitz, Uber algebraische Gebilde mit eindeutigen Transformationen in sich, Math. Ann., 41 (1893), 403-442.

[8] C. L. May, Automorphisms of compact Klein surfaces with boundary, Pacific J. Math., 59 (1975), 199-210.

[9] _ Large automorphism groups of compact Klein surfaces with boundary, Glasgow Math. J., 18 (1977), 1-10.

[10] _ Maximal symmetry and fully wound coverings, Proc. Amer. Math. Soc., 79 (1980), 23-31.

[11] M. Newman, Classification of normal subgroups of the modular group, Trans. Amer. Math. Soc., 126 (1967), 267-277. 
[12] J. J. Rotman, The Theory of Groups, Allyn and Bacon, Boston, 1973.

[13] C. H. Sah, Groups related to compact Riemann surfaces, Acta Math., 123 (1969), 13-42.

[14] W. R. Scott, Group Theory, Prentice-Hall, Englewood Cliffs, N. J., 1964.

[15] D. Singerman, Automorphisms of compact non-orientable Riemann surfaces, Glasgow Math. J., 12 (1971), 50-59.

[16] Symmetries of Riemann surfaces with large automorphism group, Math. Ann., 210 (1974), 17-32.

[17] S. E. Wilson, Operators over regular maps, Pacific J. Math., 81 (1979), 559-568.

Received July 16, 1982.

TOWSON STATE UNIVERSITY

TOWSON, MD 21204 


\title{
PACIFIC JOURNAL OF MATHEMATICS EDITORS
}

\author{
Donald BABBITT (Managing Editor) \\ University of California \\ Los Angeles, CA 90024 \\ Hugo RossI \\ University of Utah \\ Salt Lake City, UT 84112 \\ C. C. MOore and Arthur Ogus \\ University of California \\ Berkeley, CA 94720
}

J. DUGUNDJI
Department of Mathematics

University of Southern California

Los Angeles, CA 90089-1113

R. FinN and H. SAMELSON

Stanford University

Stanford, CA 94305

\section{ASSOCIATE EDITORS}
R. ARENS
E. F. BECKENBACH
B. H. NeumanN
F. WOLF
K. YosHIDA (1906-1982)

\section{SUPPORTING INSTITUTIONS}

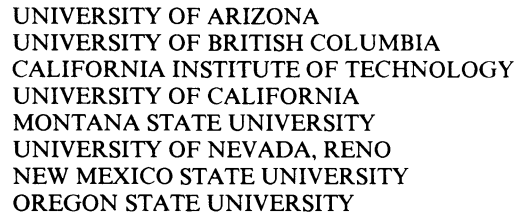

UNIVERSITY OF ARIZONA

UNIVERSITY OF BRITISH COLUMBIA

CALIFORNIA INSTITUTE OF TECHNOLOGY

UNIVERSITY OF CALIFORNIA

MONTANA STATE UNIVERSITY

UNIVERSITY OF NEVADA, RENO

NEW MEXICO STATE UNIVERSITY

OREGON STATE UNIVERSITY

\author{
UNIVERSITY OF OREGON \\ UNIVERSITY OF SOUTHERN CALIFORNIA \\ STANFORD UNIVERSITY \\ UNIVERSITY OF HAWAII \\ UNIVERSITY OF TOKYO \\ UNIVERSITY OF UTAH \\ WASHINGTON STATE UNIVERSITY \\ UNIVERSITY OF WASHINGTON
}

The Supporting Institutions listed above contribute to the cost of publication of this Journal, but they are not owners or publishers and have no responsibility for its content or policies.

Mathematical papers intended for publication in the Pacific Journal of Mathematics should be in typed form or offset-reproduced (not dittoed), double spaced with large margins. Please do not use built up fractions in the text of the manuscript. However, you may use them in the displayed equations. Underline Greek letters in red, German in green, and script in blue. The first paragraph must be capable of being used separately as a synopsis of the entire paper. In particular it should contain no bibliographic references. Please propose a heading for the odd numbered pages of less than 35 characters. Manuscripts, in triplicate, may be sent to any one of the editors. Please classify according to the scheme of Math. Reviews, Index to Vol. 39. Supply name and address of author to whom proofs should be sent. All other communications should be addressed to the managing editor, or Elaine Barth, University of California, Los Angeles, California 90024.

There are page-charges associated with articles appearing in the Pacific Journal of Mathematics. These charges are expected to be paid by the author's University, Government Agency or Company. If the author or authors do not have access to such Institutional support these charges are waived. Single authors will receive 50 free reprints; joint authors will receive a total of 100 free reprints. Additional copies may be obtained at cost in multiples of 50 .

The Pacific Journal of Mathematics is issued monthly as of January 1966. Regular subscription rate: $\$ 132.00$ a year (6 Vol., 12 issues). Special rate: $\$ 66.00$ a year to individual members of supporting institutions.

Subscriptions, orders for numbers issued in the last three calendar years, and changes of address should be sent to Pacific Journal of Mathematics, P.O. Box 969, Carmel Valley, CA 93924, U.S.A. Old back numbers obtainable from Kraus Periodicals Co., Route 100, Millwood, NY 10546.

The Pacific Journal of Mathematics ISSN 0030-8730 is published monthly by the Pacific Journal of Mathematics at P.O. Box 969, Carmel Valley, CA 93924. Application to mail at Second-class postage rates is pending at Carmel Valley, California, and additional mailing offices. Postmaster: Send address changes to Pacific Journal of Mathematics, P. O. Box 969, Carmel Valley, CA 93924.

PUBLISHED BY PACIFIC JOURNAL OF MATHEMATICS, A NON-PROFIT CORPORATION 


\section{Pacific Journal of Mathematics}

\section{Vol. 111, No. $2 \quad$ December, 1984}

Berndt Brenken, Representations and automorphisms of the irrational

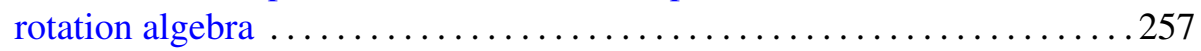

Harold George Diamond, A number theoretic series of I. Kasara . . . . . . 283

Rolf Farnsteiner, On the structure of simple-semiabelian Lie algebras . . . . 287

Guillermo Grabinsky, Poisson process over $\sigma$-finite Markov chains . ......301

Derbiau Frank Hsu and A. Donald Keedwell, Generalized complete

mappings, neofields, sequenceable groups and block designs. I . . . . . 317

William H. Julian and Fred Richman, A uniformly continuous function on

$[0,1]$ that is everywhere different from its infimum $\ldots \ldots \ldots \ldots . \ldots 33$

D. H. Lehmer and Emma Lehmer, The sextic period polynomial .........341

E. Maluta, Uniformly normal structure and related coefficients ...........357

Coy Lewis May, The species of bordered Klein surfaces with maximal

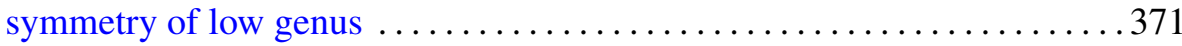

Louis Jackson Ratliff, Jr., On asymptotic prime divisors . . . . . . . . . . . 395

Norbert Riedel, Disintegration of KMS-states and reduction of standard von

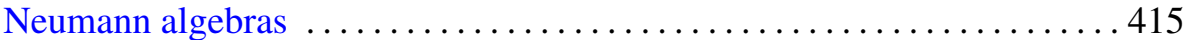

Richard Gordon Swan, $n$-generator ideals in Prüfer domains ...........433

Vilmos Totik, An interpolation theorem and its applications to positive

operators .................................. 447

Richard Vrem, Hypergroup joins and their dual objects 